\title{
Beamforming Analysis on a Broadband Antenna Array for the Galileo System
}

\author{
E. S. Neves* , M. V. T. Heckler, A. A. Selga and A. Dreher \\ German Aerospace Center (DLR), Institute of Communications and Navigation, \\ Oberpfaffenhofen, D-82234 Wessling, Germany
}

\section{Introduction}

Future navigation services provided by the upcoming Galileo satellite system will require corresponding improvements on the navigation receiving systems. The first part of any navigation receiving system is the antenna or antenna terminal. In order to obtain the best possible navigation accuracy, exploiting the full capabilities offered by this new system, design of state-of-the-art antenna systems must be pursuit.

In this work, beamforming analyses on a broadband antenna array designed for the whole Galileo frequency band [1], [2] are shown. The beamforming software [3] was developed by the DLR antenna group and employs a combination of an optimized Chebychev pattern [4] with the Sample Matrix Inversion (SMI) algorithm [5].

This paper begins with a description of the antenna array used for the simulations and its antenna element, and then the construction concepts are presented and commented. Finally, the results of the beamforming analyses are shown and discussed.

\section{Antenna Array Setup}

The single element for the Galileo antenna array [1], [2] consists of a circular microstrip patch antenna fed by means of capacitive coupling. The element has been designed with an integrated feeding system composed of a $180^{\circ}$ hybrid (rat-race) and two $90^{\circ}$ hybrids in order to generate a progressive phase shift of 90 degrees at the four feeding points. A schematic of the antenna element as well as a first prototype are shown in Fig. 1.

The impedance bandwidth of this element is approximately $31 \%$ (full Galileo band) in which the axial ratio is below $3 \mathrm{~dB}$ for elevation angles from $90^{\circ}$ down to $30^{\circ}$.

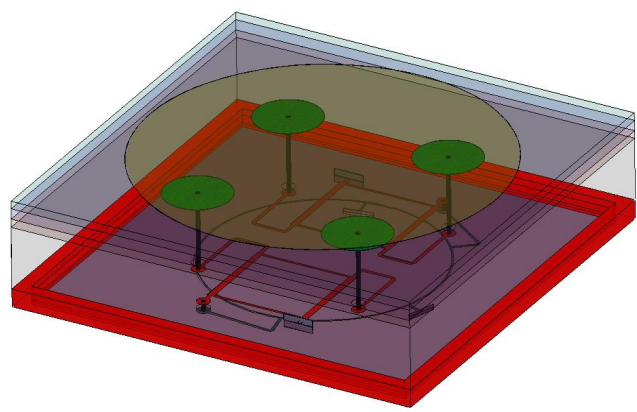

(a)

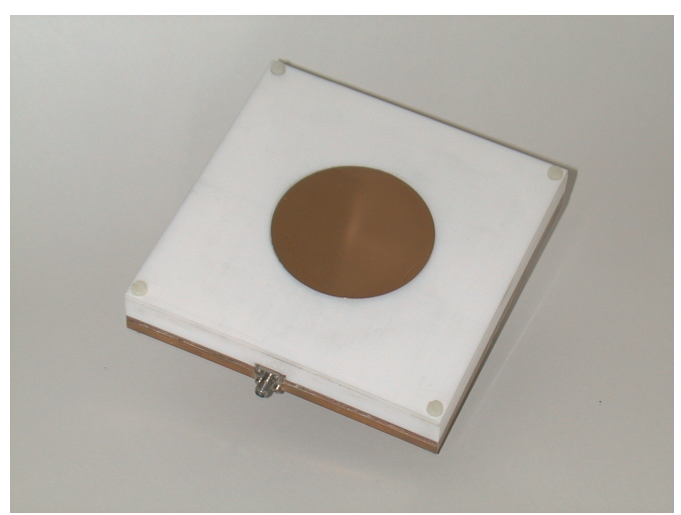

(b)

Fig. 1. Single element (a) schematic and (b) prototype. 
Fig. 2 shows the antenna array schematic in a perspective and a side view. The array size is $4 \times 4$ elements and it is mounted using a modular concept, i.e. each antenna element is a module itself. Based on the capabilities of a $4 \times 4$ antenna array using the element described above, a set of desired specifications was made as follows:

Table I. Antenna array specifications

\begin{tabular}{|l|l|}
\hline \multicolumn{1}{|c|}{ Parameter } & \multicolumn{1}{c|}{ Specification } \\
\hline Bandwidth & $1164 \mathrm{MHz}$ to $1591 \mathrm{MHz}(31 \%)$ \\
\hline Return loss & $-10 \mathrm{~dB}$ min \\
\hline Polarization & RHCP \\
\hline Azimuth scanning & $360^{\circ}$ \\
\hline Elevation scanning & from $30^{\circ}$ to $90^{\circ}$ (from $0^{\circ}$ to $30^{\circ}$ desired $)$ \\
\hline Gain & $10 \mathrm{dBi}$ min.over all scan angles $\left(30^{\circ}\right.$ to $90^{\circ}$ elevation) \\
\hline Axial ratio & $3 \mathrm{~dB}$ min. over all scan angles $\left(30^{\circ}\right.$ to $90^{\circ}$ elevation) \\
\hline Cross-polarization & $15 \mathrm{~dB}$ minimum, $25 \mathrm{~dB}$ or better is desirable \\
\hline
\end{tabular}

The overall antenna array surface is $38 \mathrm{~cm} \times 38 \mathrm{~cm}$, since each module measures $95 \mathrm{~mm} \times 95 \mathrm{~mm}$. The feeding of each element is performed by means of phase-stable coaxial cables. The adopted mechanical concept provides flexibility to the array prototype, allowing real time electronic steering as well as passive switched beamforming tests on the same platform by means of switching modules. In addition, any part of the antenna array may be easily tested isolated from the others.

(a)

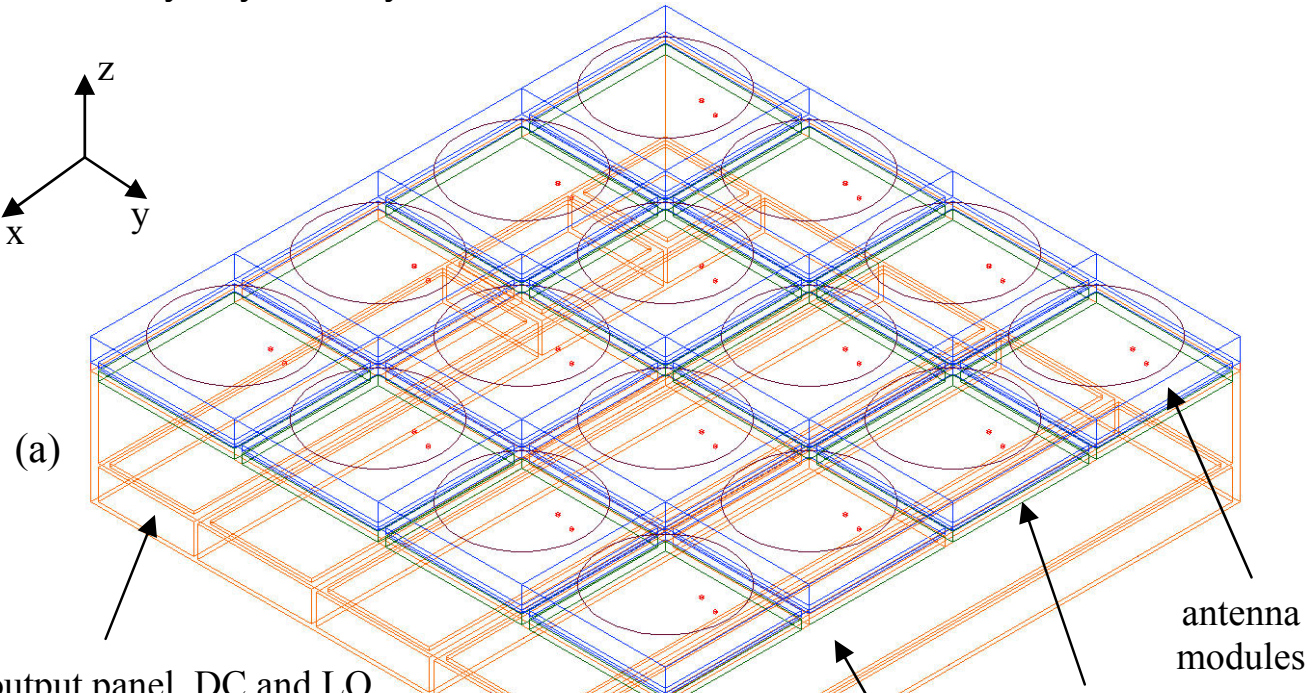

output panel, DC and LO modules, power divider networks for calibration and LO

(b)

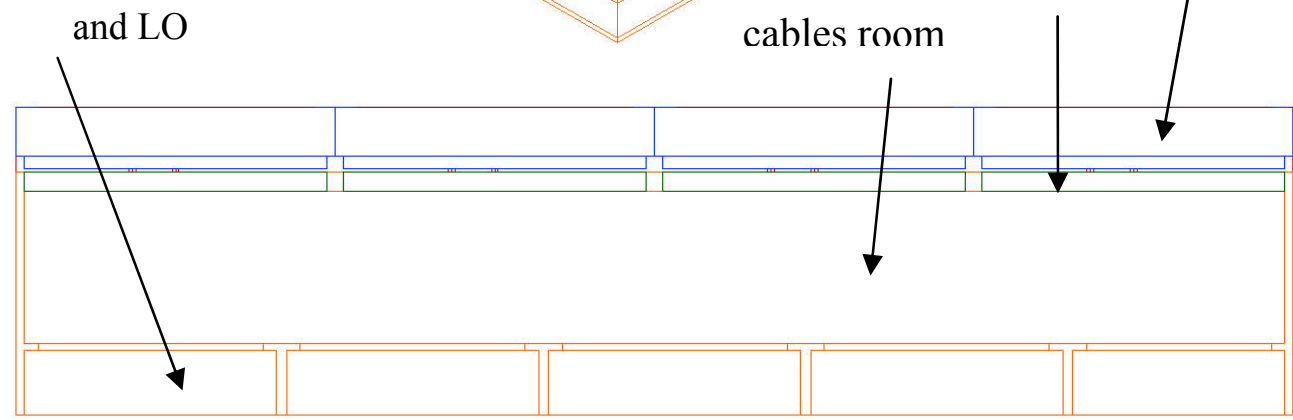

Fig. 2. Antenna array frame structure. (a) Perspective view. (b) Side view. 


\section{Beamforming Simulation Results}

In order to verify the array performance, simulations have been carried out for different beamforming conditions and frequencies. The computations have been performed using a simplified model of the antenna to suit the available computational resources. It did not consider the feeding system under each array element $\left(180^{\circ}\right.$ and $90^{\circ}$ hybrids). Therefore, the vias feeding the four plates shown in Fig. 1(a) have been directly fed with the appropriate amplitudes and phases.

Beamforming simulations including mutual coupling have been performed. For this purpose, the radiation characteristics for each array element were determined using the Planar EM Simulator from Ansoft Designer ${ }^{\circledR}$. A decoupling algorithm was applied according to the procedure described in [3]. The dielectric layers and ground plane were considered infinite in extension. Further details related to these simulations will be given at the symposium.

Since the array is designed to operate at the entire Galileo band, computations have been performed for frequencies located at the lower and upper parts of it. The first observable effect when working with a broad band is that the directivity, as well as the side lobe level (SLL) related to the main lobe, may vary significantly from the lower to the upper frequencies. For instance, when pointing the main beam to broadside and without applying any SLL suppression, a directivity of $15.65 \mathrm{dBi}$ is observed at $1.19 \mathrm{GHz}$ in contrast with $17.98 \mathrm{dBi}$ at $1.57 \mathrm{GHz}$. One reason for that is the electrical inter-element spacing, which is $0.38 \lambda_{0}$ for the first and $0.5 \lambda_{0}$ for the second frequency respectively. Moreover, the simulated gain for an isolated element in the boresight decreases from the upper to the lower part of the frequency band.

Simulations pointing the main beam to an azimuth angle of $45^{\circ}$ and different elevation angles with SLL suppression of $20 \mathrm{~dB}$ were performed. With this setup, the main beam could only be steered down to an elevation angle of $47.5^{\circ}$ at $1.19 \mathrm{GHz}$ due to the physical characteristics of the array. The calculated directivity in this case was $14.09 \mathrm{dBi}$. All lobe levels are $20 \mathrm{~dB}$ under the main beam, as shown in Fig. 3. Under these conditions, the directivity at an elevation of $30^{\circ}$, the minimum acceptable according to the specifications, is estimated to be $12.12 \mathrm{dBi}$. At $1.57 \mathrm{GHz}$, the main beam could be steered down to an elevation angle of $40^{\circ}$ with a directivity of $15.99 \mathrm{dBi}$ considering the same SLL suppression and azimuth angle as in the former case. However, two grating lobes appear with levels greater than $-20 \mathrm{~dB}$, as it can be seen in Fig. 4 .

The ratio between the $E_{\text {right }}$ and $E_{\text {left }}$ components, observed in Fig. 3 and Fig. 4, shows a reasonably good axial ratio, below $5 \mathrm{~dB}$, for elevation angles down to $30^{\circ}$.

\section{Conclusions}

Beamforming simulations for a broadband antenna array have been presented in this work. The results showed that the radiation characteristics, especially in terms of directivity and grating lobes excitation, may present significant variations within a broad frequency band demanding careful array design.

Grating lobe excitation occurred in both frequencies simulated. In the $1.19 \mathrm{GHz}$ case, the side lobe levels are all below $20 \mathrm{~dB}$ the main lobe. However, for other simulated beamforming conditions where the electrical inter-element spacing is bigger, lobes with higher levels have been excited.

Finally, a reasonable performance in terms of axial ratio was achieved. 


\section{References}

[1]E. S. Neves, A. Dreher, and P. De Vita, "Smart antenna array for Galileo and GPS applications," $2^{\text {nd }}$ ESA Workshop on Satellite Navigation User Equipment Technologies, Noordwijk, The Netherlands, 8 pages on CD-ROM, Dec. 2004.

[2] E. S. Neves, P. De Vita, and A. Dreher, "Modular Smart antenna array for GPS and Galileo Applications," The European Navigation Conference ENC-GNSS, Munich, Germany, 12 pages on CD-ROM, Jul. 2005.

[3] M. Clergeaud and M. Thiel, "Algorithms for beamforming and beamsteering of adaptive antennas for satellite communications and navigation," Internal Report, DLR-IB 554-04/01, DLR, Germany, 2003.

[4]F.-I. Tseng and D. K. Cheng, "Invariant scannable planar arrays with an invariant sidelobe level," Proc. IEEE, vol. 56, no. 11, pp. 1771-1778, Nov. 1998.

[5]I. S. Reed, J. D. Mallet and L. E. Brennan, "Rapid convergence rate in adaptive arrays," IEEE Trans. Aerosp. Electron. Syst., vol. 10, pp. 853-863, Nov. 1974.
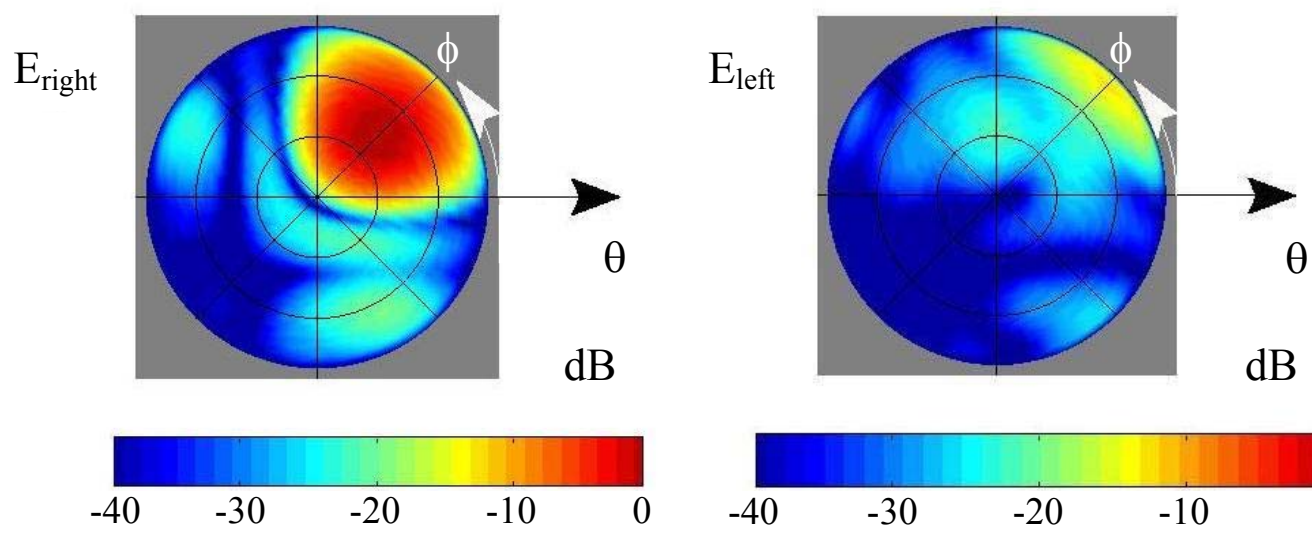

Fig. 3. Normalized Fields radiated by the antenna array at $1.19 \mathrm{GHz}$.
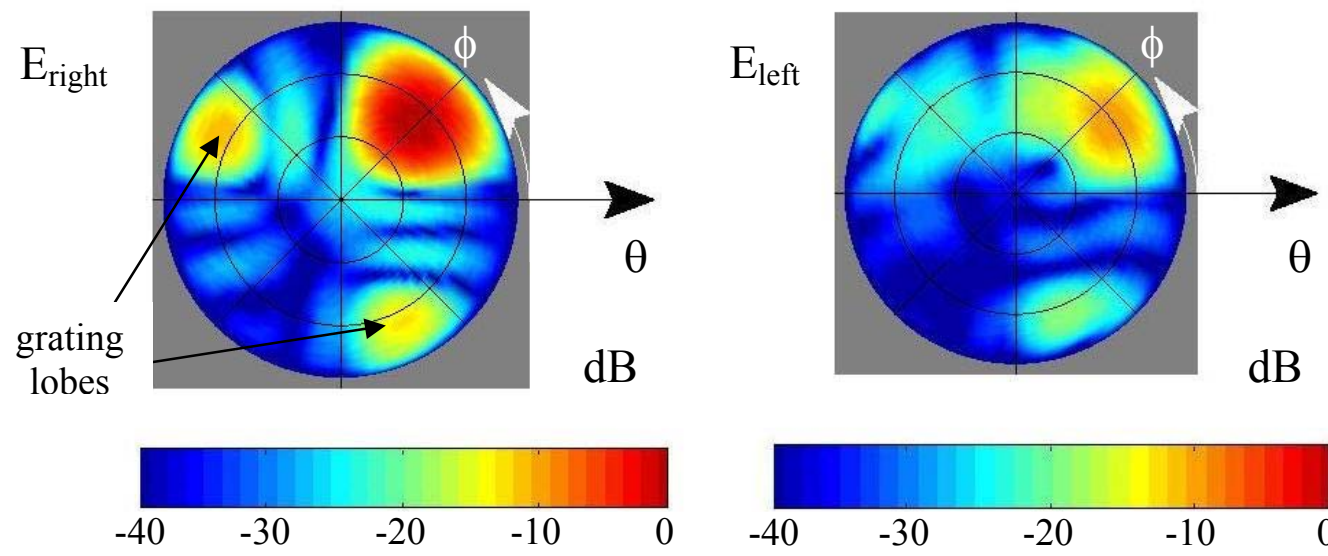

Fig. 4. Normalized Fields radiated by the antenna array at $1.57 \mathrm{GHz}$. 